Mitigating End Effects in the Dynamic Vehicle Allocation Model

Author(s): Richard E. Hughes and Warren B. Powell

Source: Management Science, Vol. 34, No. 7 (Jul., 1988), pp. 859-879

Published by: INFORMS

Stable URL: http://www.jstor.org/stable/2632300

Accessed: 23/08/2011 17:20

Your use of the JSTOR archive indicates your acceptance of the Terms \& Conditions of Use, available at http://www.jstor.org/page/info/about/policies/terms.jsp

JSTOR is a not-for-profit service that helps scholars, researchers, and students discover, use, and build upon a wide range of content in a trusted digital archive. We use information technology and tools to increase productivity and facilitate new forms of scholarship. For more information about JSTOR, please contact support@jstor.org. 


\title{
MITIGATING END EFFECTS IN THE DYNAMIC VEHICLE ALLOCATION MODEL*
}

\author{
RICHARD E. HUGHES AND WARREN B. POWELL \\ Department of Industrial and Operations Engineering, University of Michigan, \\ Ann Arbor, Michigan 48109 \\ Department of Civil Engineering and Operations Research, School of Engineering/Applied \\ Science, Princeton University, Princeton, New Jersey 08544
}

\begin{abstract}
A class of network models developed for optimizing the use of a fleet of vehicles can be viewed as time-staged optimization models. Such models which have linear objective functions suffer particularly from end effects, the distortions introduced by employing a finite planning horizon. This paper brings together the end effects literature and the vehicle allocation literature to find a finite linear programming problem that gives an approximation to the first stage optimal solution of the infinite model. In addition, we develop another method of approximation and show that both methods can be formulated as generalized networks. Numerical results are presented for each method for comparison.

(GENERALIZED NETWORKS; VEHICLE PLANNING)
\end{abstract}

\section{Introduction}

Many transportation companies face the problem of deciding how to manage their vehicle fleet over time. That decision process is particularly important for companies that have full control over their vehicle movements on a real-time basis, and that are not bound by a service schedule. Truckload motor carriers, sea container companies, and rental car services all fall into this category. Each industry is characterized by a large, spatially distributed fleet of vehicles which must be moved to handle current demands or repositioned (moved empty) to handle known or anticipated demands at future points in time. Since demands vary over time as well as space, the problem is referred to as the dynamic vehicle allocation problem (DVA).

In the truckload industry, for example, a carrier agrees with a customer to carry its goods from one location to another without sharing the truck with any other customer. Thus the company commits trucks to servicing a customer for a period of time. At the end of that time, the decision of what to do with the truck arises again. The decisions faced by a carrier include which loads should be accepted on a given day, which trailers should be moved empty to another region and which trailers should be held in a region until a later time.

The sea container industry faces a similar situation. Containers move according to the needs of individual customers and according to management decisions about empty container movements. Tradeoffs occur between demand for a container now that would not be very profitable and future demand for the container either in the same location or some other place that would be more profitable. The choice then is to either allocate the container to meet the present demand, keep it as inventory, or move it empty somewhere else.

In the car and truck rental industries, customers often rent a vehicle at one location and "return" it to the company at a different location. Hence the same kinds of decisions must be made about what to do with the cars. Clearly the spatial structure of this industry is discrete. A car rental company has a finite number of car lots to which

\footnotetext{
* Accepted by Alexander H. G. Rinnooy Kan; received November 1985. This paper has been with the authors 7 months for 2 revisions.
} 
cars can be returned and from which they can be rented. Moreover, the truckload and sea container industries can be modelled similarly by aggregating the service area into regions. Thus all three models have a discrete number of locations, and time can be considered as discrete since vehicle allocation decisions might be made daily.

Typically a company that would use a DVA model faces the situation where it has full control over each of its vehicle's movements and each vehicle serves only one customer at a time. At a particular time a vehicle can either be assigned to fulfill a customer's demand for transportation services from point $a$ to $b$, it can move empty, or it can remain in place to take advantage of future demand where it is. Each choice has a certain impact in terms of immediate costs and revenues derived, as well as downstream impacts resulting from having a vehicle in a new location. By not considering these future impacts, the profit a vehicle will earn carrying a load into a region can be significantly reduced if the vehicle must then be moved empty out of the region.

Decisions made today, then, must anticipate future opportunities. An important assumption made in this research is that future revenue generating opportunities are either known or can be reasonably predicted based on previous historical activities. In addition, the assumption is made that activities follow a cyclical pattern (reflecting, for example, a weekly cycle in activity levels), implying that in principle at least predictions of future demands can be made indefinitely into the future. Incorporating such information would require solving an infinitely large linear program, however, so the decision maker must truncate the planning horizon-the time beyond which future demand information is ignored-to a finite value. The result of ignoring future demand can potentially result in serious distortions of the solution. This distortion is known as an "end effect" since it comes from an artificial end of the planning horizon.

To illustrate end effects, consider a United States truckload motor carrier. Suppose one of its regions is Alaska, which generally has a large quantity of goods being trucked in and little trucked out. Clearly the profit and deadheading costs from the continental United States to Alaska are large. In an infinite horizon model there would be a disincentive to taking shipments to Alaska because it is very likely that the truck would have to come back empty. If the planning horizon were truncated to a finite length, the optimal solution might send too many trucks to Alaska because the "deadhead" penalty has been removed by the truncation. Moreover, in prior periods the model would allocate trucks to take advantage of the last period opportunity. So it is easy to see how truncating an infinite horizon DVA model to a finite horizon one introduces end effects.

The DVA model has its origins in Leddon and Wrathall (1967), Potts (1970), White (1972), White and Bomberault (1969). Jordan and Turnquist (1983) and Powell (1986) extend this work to the case of stochastic demands. These works introduce the basic network structure used in the DVA model formulation considered here, which is summarized more clearly in Powell et al. (1984). Currently the DVA models being used lack a theoretically sound basis for reducing the severity of the end effects. There is, however, an extensive literature on time-staged optimization models. Since time-staged optimization models often occur in economic and capacity expansion models, it is in these areas that the end effects literature has developed. Hopkins (1971) addresses end effects in the capacity expansion context. Manne (1970) considers end effects in the economic development context. Grinold (1977, 1983a, b) and Grinold and Hopkins (1973) also provide results for reducing end effects in time-staged optimization models. It is the four papers of Grinold and Grinold and Hopkins that are directly relevant to the DVA model.

The paper is organized as follows. $\S 2$ reviews the basic formulation of the dynamic vehicle allocation model. $\S 3$ then presents several approaches for mitigating end effects by solving specific finite linear programs that can be represented as generalized net- 
works. Finally, $\S 4$ presents the results from an extensive set of numerical experiments where various rigorous as well as naive approximations are used to solve the DVA. These techniques are evaluated by comparing them to a "brute force" solution which solved a very large network problem extending well into the future.

\section{Basic DVA Model}

The DVA model assumes that both the spatial and temporal aspects of the situation can be discretized. For example, a trucking company can divide its service area into regions and make dispatch decisions at times $\tau_{0}, \tau_{1}, \tau_{2}, \ldots$, which are also called periods $0,1,2, \ldots$ In transportation, these time periods are often evenly spaced in time, although they need not be. In the trucking industry, for example, a typical time period would be 24 hours. Consider the decision of how to use a truck in a region in a particular time period $i$, which is made at time $\tau_{i}$. The company must choose whether to (1) assign the truck to a load (if one exists), (2) move the truck empty to another region, or (3) keep the truck in the same region to take advantage of future demand originating in that region.

In the DVA model considered here, the future demand for vehicles is assumed to be known with certainty. While not entirely accurate, the assumption is particularly useful since it yields a linear objective function for the mathematical programming problem. The DVA model can thus be written as a linear program, where the constraints are merely the conservation of vehicles in each region at each time period of the planning horizon, plus the upper bounds on the loaded vehicle movements which are equal to the projected demands. Let $\mathrm{C}$ be the set of regions and $N$ be the number of time periods that constitute the planning horizon. Then the DVA model is given by:

subject to

$$
\max \Pi=\sum_{i \in C} \sum_{j \in C} \sum_{n=1}^{N} \alpha^{n}\left[r_{i j} F_{i j, n}-d_{i j} E_{i j, n}\right]
$$

$$
\begin{gathered}
\sum_{i \in C}\left[F_{i j, n-t_{i j}}+E_{i j, n-t_{i j}}\right]+R_{i, n}=\sum_{k \in C}\left[F_{j k, n}+E_{j k, n}\right] \quad \forall j, n \\
0 \leq F_{i j, n} \leq D_{i j, n}, \quad \forall i, j, n, n=1, \ldots, N, \quad \text { where }
\end{gathered}
$$

$E_{i j, n}=$ the flow of empties going from region $i$ to region $j$, leaving on period $n$,

$F_{i j, n}=$ the flow of full vehicles going from region $i$ to region $j$, leaving on period $n$,

$t_{i j}=$ travel time (measured in periods) required to go from region $i$ to region $j$, where $t_{i j}=1,2, \ldots$,

$d_{i j}=$ cost to send an empty vehicle from region $i$ to region $j$ (deadhead cost),

$r_{i j}=$ net revenue received from sending a full vehicle from region $i$ to region $j$,

$D_{i j, n}=$ demand for vehicles going from region $i$ to region $j$, leaving on period $n\left(D_{i j, n}\right.$ $=0,1,2, \cdots)$,

$R_{i, n}=$ supply of vehicles entering the system for the first time at city $i$ on period $n$ (negative if vehicles leave the system),

$\alpha=$ discount factor.

Note that each decision variable in (1) originates at a node and terminates at another. Clearly the DVA model can be formulated as a network by making each region at each time period a node. Constraint (2) represents the conservation of flow restriction at each node. An example with two regions and one-period vehicle movements is depicted in Figure 1. Note that there are pairs of arcs between nodes: one arc is for vehicles moving full; the other, for vehicles deadheading. Equation (3) specifies the upper bounds on the full vehicle movement arcs to be the demand for vehicles going from $i$ to $j$ leaving in period $n$. Moreover, the basis of this network model is unimodular since 
there are no gains or losses on the arcs. It is the pure network structure which allows the use of very efficient network simplex codes to solve DVA models.

In equation (1), each successive time period is discounted again by $\alpha$. Typically, however, time periods would be grouped together in stages, where all activities in a given stage are discounted equally. For example, seven one-day time periods might represent a single stage. In addition, the first few time periods are often grouped into an initial transient stage of arbitrary length while the rest of the planning horizon is made up of a single stationary stage that is repeated continuously. The transient stage might incorporate real-time information about actual and forecasted demands, while the stationary stage would consist entirely of demand forecasts based on historical data.

Obviously an important parameter in this model is the choice of the discount factor $\alpha$. One option of course would be based on the time value of money, leading to a choice of $\alpha$ very close to unity. This option does not accurately discount future activities since in reality there is some uncertainty associated with the forecasts of demands for vehicles. An alternative, then, is to choose a considerably smaller discount factor (say 0.6 or 0.7 ) as a somewhat ad hoc approach for incorporating uncertainty.

Although Figure 1 indicates an infinite time dimension, in practice a finite number of time periods are considered. Therefore a supersink is added beyond the planning horizon. Uncapacitated arcs representing trucks that reach their destinations beyond the planning horizon terminate at the supersink. A cost is put on each arc terminating at the supersink which is referred to as the regional penalty of the region in which the arc originates. In the trucking example of the introduction, the arcs leaving the nodes corresponding to Alaska and terminating at the supersink would have a large positive penalty. The state of the art in solving the DVA model consists of heuristics for finding these regional penalties. These methods, however, lack theoretical support. Applying the time-staged optimization model end effects literature can provide that support.

\section{Methods for Reducing End Effects in the DVA Model}

One way of tackling the end effects problem is to find a finite sized mathematical programming problem which approximates the infinite horizon problem for a given number of periods into the future. This section presents three such problem formulations. The first, the dual equilibrium method of Grinold (1983a), is shown to exhibit special structure when applied to the DVA model. The second method, termed here the generalized summation approach, is closely related to the dual equilibrium method but uses a slightly different method for aggregating future time periods. Finally, the results of Grinold and Hopkins (1973) are applied to the DVA model to give the Leontief approximation.

\section{Dual Equilibrium Approximation}

The mathematical programming problem considered in Grinold (1983a) is given by

subject to

$$
\min u=f_{0}\left(\mathbf{x}_{0}\right)+\sum_{t=1}^{\infty} \alpha^{t} f\left(\mathbf{x}_{t}\right)
$$

$$
\begin{array}{ll}
\mathbf{A}_{\mathbf{0}} \mathbf{x}_{\mathbf{0}} & =\mathbf{b}_{\mathbf{0}}, \\
\mathbf{H}_{\mathbf{1}} \mathbf{x}_{\mathbf{0}}+\mathbf{A} \mathbf{x}_{\mathbf{1}} & =\mathbf{b}_{\mathbf{1}}, \\
\mathbf{H}_{2} \mathbf{x}_{\mathbf{0}}+\mathbf{K}_{\mathbf{1}} \mathbf{x}_{\mathbf{1}}+\mathbf{A} \mathbf{x}_{\mathbf{2}} & =\mathbf{b}_{\mathbf{2}}, \\
\mathbf{H}_{\mathbf{3}} \mathbf{x}_{\mathbf{0}}+\mathbf{K}_{\mathbf{2}} \mathbf{x}_{\mathbf{1}}+\mathbf{K}_{\mathbf{1}} \mathbf{x}_{\mathbf{2}}+\mathbf{A} \mathbf{x}_{\mathbf{3}} & =\mathbf{b}_{\mathbf{3}},
\end{array}
$$




$$
\begin{array}{ll}
\mathbf{H}_{\mathbf{4}} \mathbf{x}_{\mathbf{0}}+\mathbf{K}_{\mathbf{3}} \mathbf{x}_{\mathbf{1}}+\mathbf{K}_{\mathbf{2}} \mathbf{x}_{\mathbf{2}}+\mathbf{K}_{\mathbf{1}} \mathbf{x}_{\mathbf{3}}+\mathbf{A} \mathbf{x}_{\mathbf{4}} & =\mathbf{b}_{\mathbf{4}}, \\
: & \\
\mathbf{H}_{\mathbf{t}} \mathbf{x}_{\mathbf{0}}+\mathbf{K}_{\mathbf{t}-\mathbf{1}} \mathbf{x}_{\mathbf{1}}+\mathbf{K}_{\mathbf{t}-\mathbf{2}} \mathbf{x}_{\mathbf{2}}+\cdots+\mathbf{A} \mathbf{x}_{\mathbf{t}}=\mathbf{b}_{\mathbf{t}},
\end{array}
$$

$$
\mathbf{x}_{\mathbf{0}}, \mathbf{x}_{\mathbf{t}} \geq \mathbf{0}, \quad t \geq 1
$$

where $f$ and $f_{0}$ are lower semicontinuous functions. The decision variables contained in $\mathbf{x}_{0}$ form the "transient stage" while $\mathbf{x}_{t}, t \geq 1$, form the "stationary" part of the mathematical program.

The dual equilibrium method of Grinold (1983a) aggregates the decision variables of the stationary section of (5) to give a finite linear program. The constraints for time $t$ $\geq 1$ are weighted by $\alpha^{t-1}(1-\alpha)$, where $\sum_{t=1}^{\infty} \alpha^{t-1}(1-\alpha)=1$. Summing over $t$ gives

$$
\begin{aligned}
(1-\alpha)\left[\sum_{t=1}^{\infty} \alpha^{t-1} \mathbf{H}_{t}\right] x_{0}+\mathbf{A}(1-\alpha) & \sum_{t=1}^{\infty} \alpha^{t-1} \mathbf{x}_{\mathbf{t}} \\
& +\left[\sum_{t=1}^{\infty} \alpha^{t} \mathbf{K}_{t}\right]\left[(1-\alpha) \sum_{t=1}^{\infty} \alpha^{t-1} \mathbf{x}_{t}\right]=(1-\alpha) \sum_{t=1}^{\infty} \alpha^{t-1} \mathbf{b}_{t} .
\end{aligned}
$$

Substituting aggregate quantities gives

$$
\begin{aligned}
\mathbf{H}(\alpha) \mathbf{x}_{0}+[\mathbf{A}(\alpha)] \mathbf{x}(\alpha)=\mathbf{b}(\alpha), \quad \text { where } \\
\mathbf{b}(\alpha)=(1-\alpha) \sum_{t=1}^{\infty} \alpha^{t-1} \mathbf{b}_{\mathbf{t}} \\
\mathbf{H}(\alpha)=(1-\alpha) \sum_{t=1}^{\infty} \alpha^{t-1} \mathbf{H}_{\mathbf{t}} \\
\mathbf{A}(\alpha)=\mathbf{A}+\sum_{t=1}^{\infty} \alpha^{t} \mathbf{K}_{\mathbf{t}} \\
\mathbf{x}(\alpha)=(1-\alpha) \sum_{t=1}^{\infty} \alpha^{t-1} \mathbf{x}_{\mathbf{t}}
\end{aligned}
$$

Similarly, the objective function can be approximated by aggregating the decision variables. If $f$ is a convex and lower semicontinuous function,

$$
\sum_{t=1}^{\infty} \alpha^{t} f\left(\mathbf{x}_{t}\right)=\alpha\left[\sum_{t=1}^{\infty}(1-\alpha) \alpha^{t-1} f\left(\mathbf{x}_{t}\right)\right] /(1-\alpha) \geq \alpha f(\mathbf{x}(\alpha)) /(1-\alpha) .
$$

Of course, equation (12) holds with equality when $f$ is linear. Since $f\left(\mathbf{x}_{0}\right)=\mathbf{c}_{0} \mathbf{x}_{0}$ and $f\left(\mathbf{x}_{t}\right)=\mathbf{c} \mathbf{x}_{t}(t \geq 1)$ for the DVA model, the objective function can be written

$$
\sum_{t=0}^{\infty} \alpha^{t} \mathbf{c}_{t} \mathbf{x}_{t}=\mathbf{c}_{0} \mathbf{x}_{0}+\alpha \sum_{t=1}^{\infty} \alpha^{t-1} \mathbf{c} \mathbf{x}_{t}=\mathbf{c}_{0} \mathbf{x}_{0}+\alpha \mathbf{c x}(\alpha) /(1-\alpha) .
$$

Thus the dual equilibrium finite approximation problem is

$$
\min \mathbf{c}_{\mathbf{0}} \mathbf{x}_{\mathbf{0}}+\alpha \mathbf{c x}(\alpha) /(1-\alpha)
$$

subject to

$$
\begin{gathered}
\mathbf{A}_{\mathbf{0}} \mathbf{x}_{\mathbf{0}}=\mathbf{b}_{\mathbf{0}}, \\
\mathbf{H}(\alpha) \mathbf{x}_{0}+\mathbf{A}(\alpha) \mathbf{x}(\alpha)=\mathbf{b}(\alpha), \\
\mathbf{x}_{\mathbf{0}} \geq \mathbf{0}, \quad \mathbf{x}(\alpha) \geq \mathbf{0} .
\end{gathered}
$$


Note that it is not possible to reconstruct the stationary stage solution to the infinite horizon problem (4)-(5) from $\mathbf{x}(\alpha)$. However, this does not pose a problem, for the goal is to reduce the end effects in the transient stage solution. The number of columns in (15)-(17) is equal to the number of empty movement arcs plus twice the number of loaded movement arcs in the time periods aggregated into $\mathbf{x}_{0}$ and $\mathbf{x}_{1}$ of (5). The number of rows in (15)-(17) is the sum of the number of nodes and loaded $\operatorname{arcs}$ in $\mathbf{x}_{0}$ and $\mathbf{x}_{1}$ of (5).

To illustrate how the DVA model is consistent with the form of (4)-(5), consider the example presented in Figure 1. In this example each stage is defined as consisting of two time periods. That holds for the transient stage as well as for the stages of the stationary section of the model. Accordingly, $\mathbf{K}_{t}=\mathbf{H}_{t}=\mathbf{0}$ for $t>1$. It is convenient to partition the rows of $\mathbf{A}$ and $\mathbf{A}_{0}$ into two groups: $\mathbf{A}_{f}$ contains the rows for the conservation of flow equations while $\mathbf{A}_{b}$ contains the entries for the upper bounds on the loaded movement arcs. Thus,

$$
\begin{aligned}
& A_{0}=A=\left[\begin{array}{l}
A_{f} \\
A_{b}
\end{array}\right],
\end{aligned}
$$

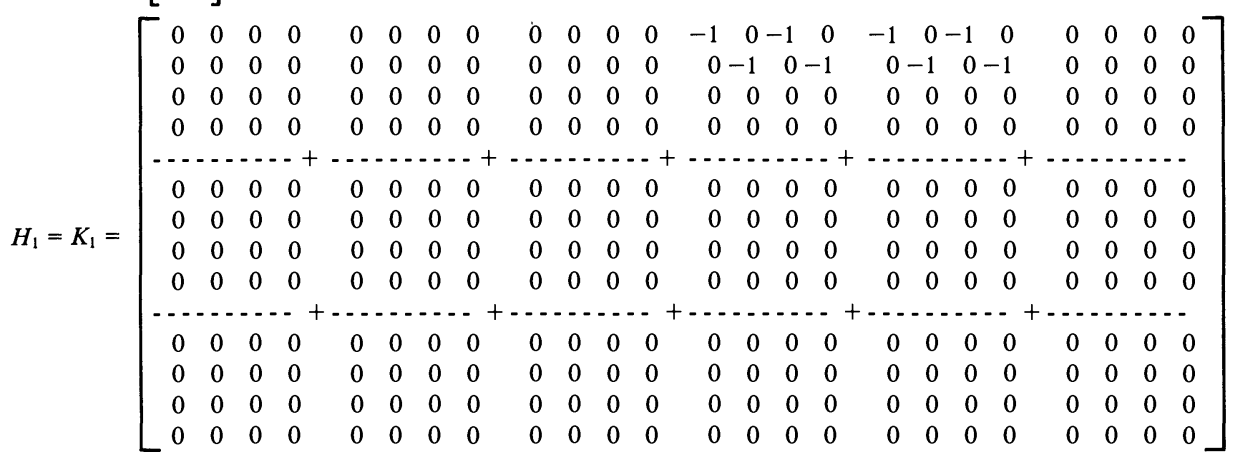

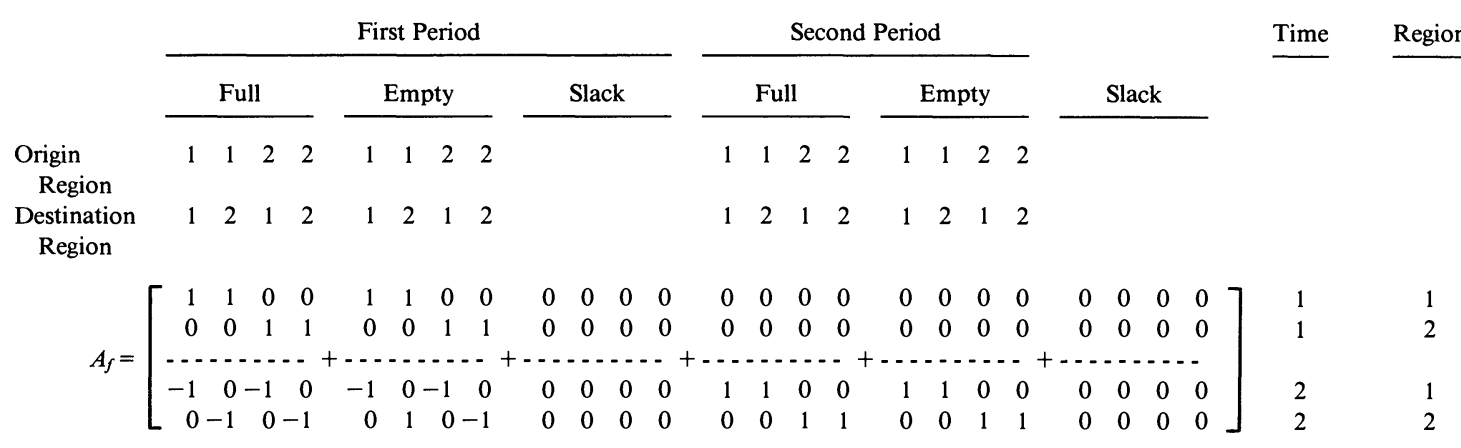

$$
A_{b}=\left[\begin{array}{cccccccccccccccccccccccc}
1 & 0 & 0 & 0 & 0 & 0 & 0 & 0 & 1 & 0 & 0 & 0 & 0 & 0 & 0 & 0 & 0 & 0 & 0 & 0 & 0 & 0 & 0 & 0 \\
0 & 1 & 0 & 0 & 0 & 0 & 0 & 0 & 0 & 1 & 0 & 0 & 0 & 0 & 0 & 0 & 0 & 0 & 0 & 0 & 0 & 0 & 0 & 0 \\
0 & 0 & 1 & 0 & 0 & 0 & 0 & 0 & 0 & 0 & 1 & 0 & 0 & 0 & 0 & 0 & 0 & 0 & 0 & 0 & 0 & 0 & 0 & 0 \\
0 & 0 & 0 & 1 & 0 & 0 & 0 & 0 & 0 & 0 & 0 & 1 & 0 & 0 & 0 & 0 & 0 & 0 & 0 & 0 & 0 & 0 & 0 & 0 \\
\hdashline 0 & 0 & 0 & 0 & 0 & 0 & 0 & 0 & 0 & 0 & 0 & 0 & 1 & 0 & 0 & 0 & 0 & 0 & 0 & 0 & 1 & 0 & 0 & 0 \\
0 & 0 & 0 & 0 & 0 & 0 & 0 & 0 & 0 & 0 & 0 & 0 & 0 & 1 & 0 & 0 & 0 & 0 & 0 & 0 & 0 & 1 & 0 & 0 \\
0 & 0 & 0 & 0 & 0 & 0 & 0 & 0 & 0 & 0 & 0 & 0 & 0 & 0 & 1 & 0 & 0 & 0 & 0 & 0 & 0 & 0 & 1 & 0 \\
0 & 0 & 0 & 0 & 0 & 0 & 0 & 0 & 0 & 0 & 0 & 0 & 0 & 0 & 0 & 1 & 0 & 0 & 0 & 0 & 0 & 0 & 0 & 1
\end{array}\right]
$$$$
\mathbf{c}_{0}=\mathbf{c}=\left[-r_{11}-r_{12}-r_{21}-r_{22} d_{11} d_{12} d_{21} d_{22} 00000\right.
$$

$$
\left.-r_{11}-r_{12}-r_{21}-r_{22} d_{11} d_{12} d_{21} d_{22} 0 \begin{array}{llll}
0 & 0 & 0
\end{array}\right]
$$




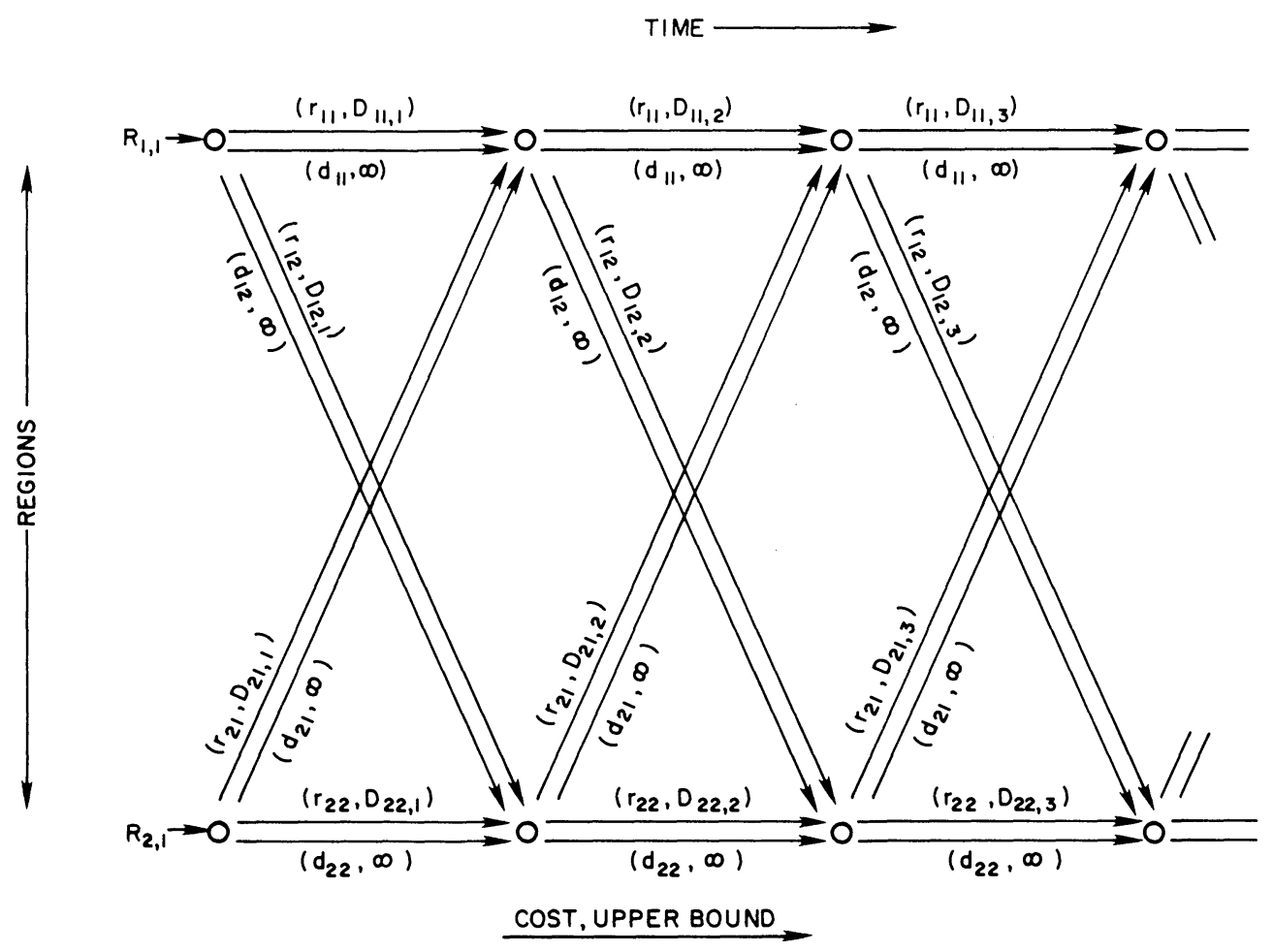

FIGURE 1. The Infinite Stage Dynamic Vehicle Allocation Problem.

and for stage $t$,

$$
\mathbf{b}_{t}=\left[\begin{array}{llllll}
R_{1,2 t} & R_{2,2 t} R_{1,2 t+1} R_{2,2 t+1} & D_{11,2 t} D_{12,2 t} D_{21,2 t} D_{22,2 t} D_{11,2 t+1} D_{12,2 t+1} D_{21,2 t+1} D_{22,2 t+1}
\end{array}\right] .
$$

Note that the rows in $H_{1}$ and $K_{1}$ consisting of all 0 's are necessary to make all matrices in (16) have the proper dimensions. $H_{1}$ and $K_{1}$ contain entries corresponding to the termination of arcs which cross between time stages.

In general, the DVA model can be written in the form of (4)-(5) by aggregating the constraints and decision variables by time stage. The lower block triangular structure of the constraints is due to the dynamic nature of the model. That is, a vehicle that departs at time $\tau_{i}$ cannot possibly finish its trip at time $\tau_{j}<\tau_{i}$. Thus when the constraints and decision variables are ordered according to time period, no arcs can move upward across the diagonal of the constraint matrix; hence the upper triangle is left full of zeros.

Grinold (1983a) requires that the quantities $\mathbf{A}(\alpha), \mathbf{H}(\alpha), \mathbf{K}(\alpha)$ and $\mathbf{b}(\alpha)$ exist. In the context of the DVA model this means that there is a cycle in the demand for vehicles, as well as a regular pattern in the possible vehicle movements. Therefore either the transportation mode being modelled must exhibit these properties in order to apply the dual equilibrium technique or it must be possible to approximate the actual situation by a model having these properties.

Not only does the DVA model satisfy the mild restrictions outlined above, as we have just seen for a two-region model, but the finite mathematical program which gives an approximation of $\mathbf{x}_{0}$, problem (14)-(17), has a very special structure. This important result is stated as a theorem about the DVA model.

THEOREM 1. Equations (15)-(17) form the conservation of flow equations for a generalized network. 
PROOF. A generalized network is characterized by a constraint matrix where each column has at most two nonzero entries, in addition to upper bounds which can be ignored. The vector $\mathbf{x}_{0}$ covers links which begin in the transient stage and end in either the transient or stationary stage. For links which begin and end in the transient stage, $\mathbf{A}_{0}$ contains a single +1 and -1 and the column of $\mathbf{H}(\alpha)$ corresponding to this link consists of all 0's. Links which end in the stationary stage are represented by $\mathrm{a}+1$ in $\mathbf{A}_{0}$ and $\mathrm{a}-\alpha$ in $\mathbf{H}(\alpha)$. We thus need to show that the matrix $\mathbf{A}(\alpha)$ has at most two nonzero entries. Recall that $\mathbf{A}(\alpha)=\mathbf{A}+\sum_{t=1}^{\infty} \alpha^{t} \mathbf{K}_{t}$. Any link moving within a single stationary stage contains $\mathbf{a}+1$ and -1 in the associated column of $\mathbf{A}$ while the corresponding column in $\sum_{t=1}^{\infty} \alpha^{t} \mathbf{K}_{t}$ is all zero. Links moving from one stationary stage to the next are represented by a single +1 in $\mathbf{A}$ and $\mathrm{a}-1$ in the column of the sequence $\left\{\mathbf{K}_{t}\right\}$.

$A(\alpha)$ and $H(\alpha)$, which represent the condensed stationary stage, is given below. The first twelve variables represent arcs which originate in the transient stage; the last twelve are for arcs originating in the stationary stage.

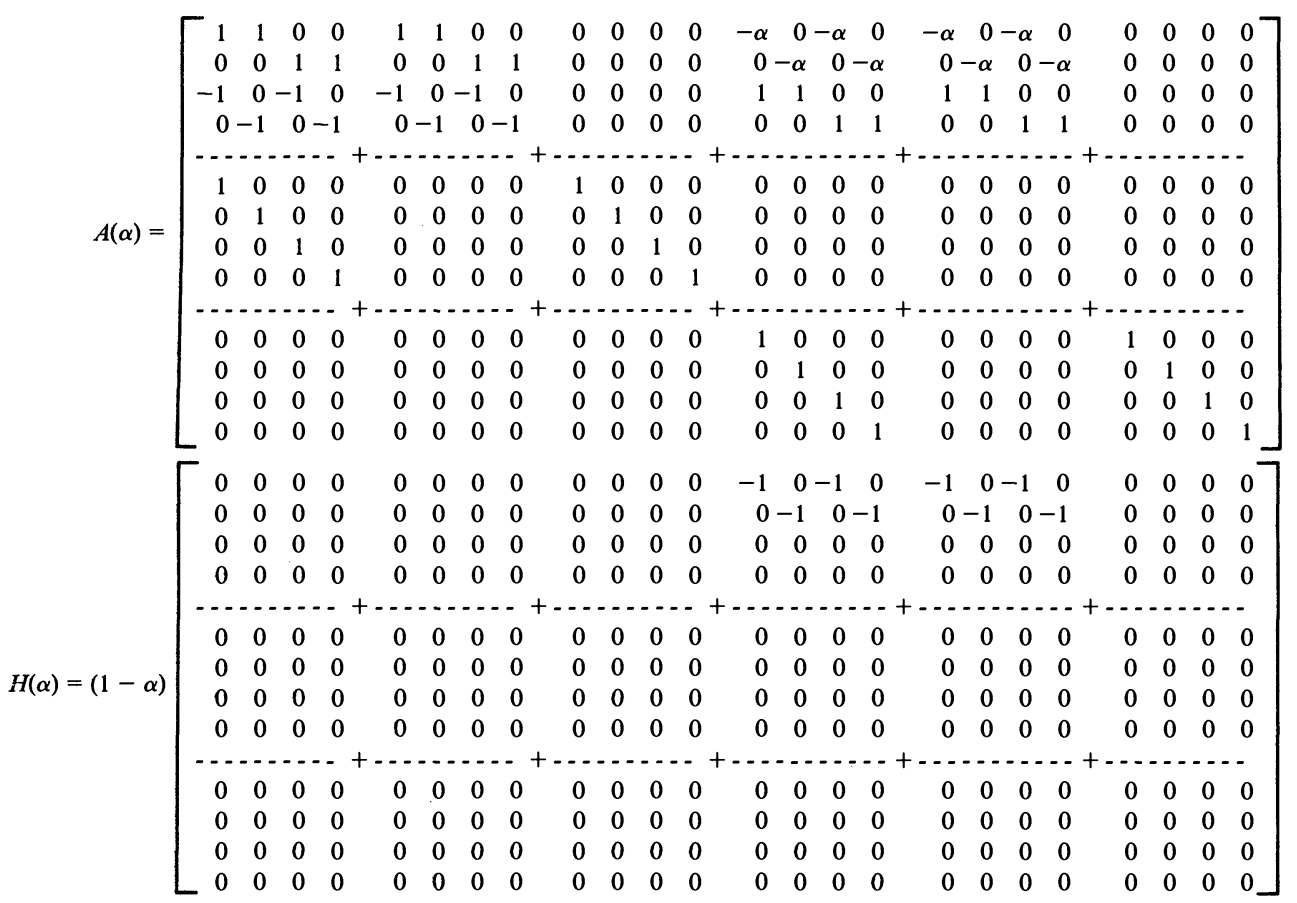

Assuming that no trucks enter or leave the system after the transient phase and that the demands are cyclical,

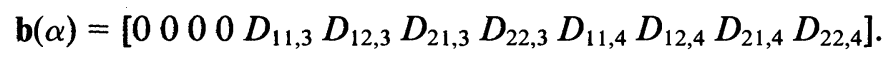

The corresponding network is given in Figure 2.

The result that (15)-(16) is a generalized network is very important, because it allows the use of specialized solution methodologies for solving generalized networks, such as Brown and McBride (1985), to find the optimal solution to (14)-(17).

\section{Generalized Summation Method}

Intuitively, the dual equilibrium technique works by taking the sum of an infinite geometric series. That is, when a unit of flow passes from the transient section to the stationary section of the finite model (see Figure 2), it enters a loop which links the end 


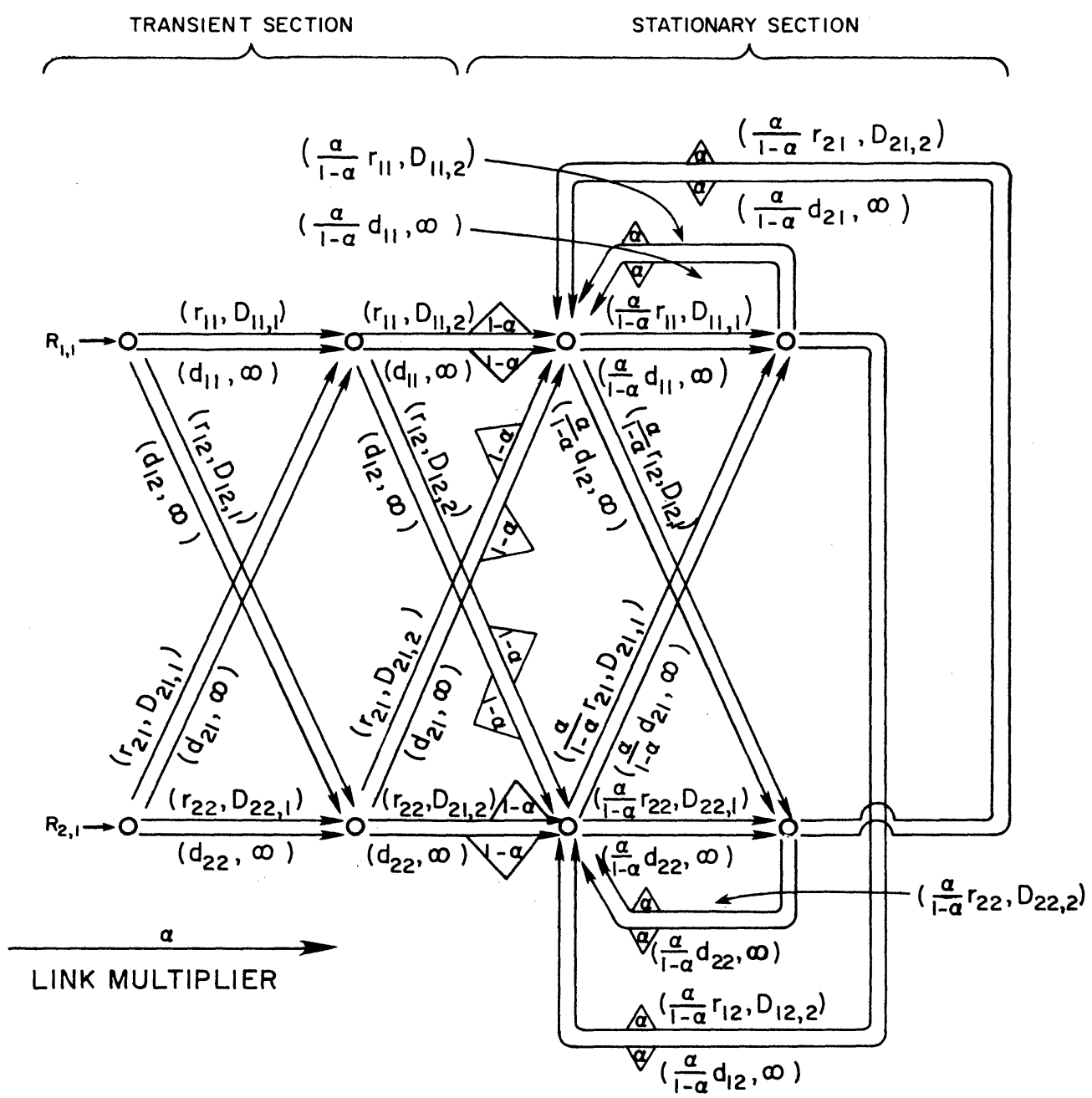

FIGURE 2. Representation of the Dual Equilibrium Technique as a Generalized Network.

of the aggregated stationary period to the beginning of the stationary period. As the flow cycles around the loop, it is factored down by $\alpha, 0<\alpha<1$, which effectively makes $\mathbf{x}(\alpha)$ the sum of an infinite geometric sequence. The generalized summation method, illustrated in Figure 3, is based on the same intuition. The generalized summation method differs from the dual equilibrium in the way the stationary period is aggregated.

The generalized summation method provides a somewhat more intuitive approach to the method of aggregating future time periods. The objective function of the infinite horizon DVA model is

$$
\sum_{t=0}^{\infty} \alpha^{t} \mathbf{c}_{t} \mathbf{x}_{t}
$$

Under the restriction that $\mathbf{c}_{i}=\mathbf{c}_{j}, i, j \geq 1$, this is

$$
\mathbf{c}_{0} \mathbf{x}_{0}+\mathbf{c} \sum_{t=1}^{\infty} \alpha^{t} \mathbf{x}_{t}
$$

The generalized summation method aggregates the stationary period into $\mathbf{x}_{g s}(\alpha)$ $=\sum_{t=1}^{\infty} \alpha^{t} \mathbf{x}_{t}$, representing a straightforward net present value of future flows. Therefore 


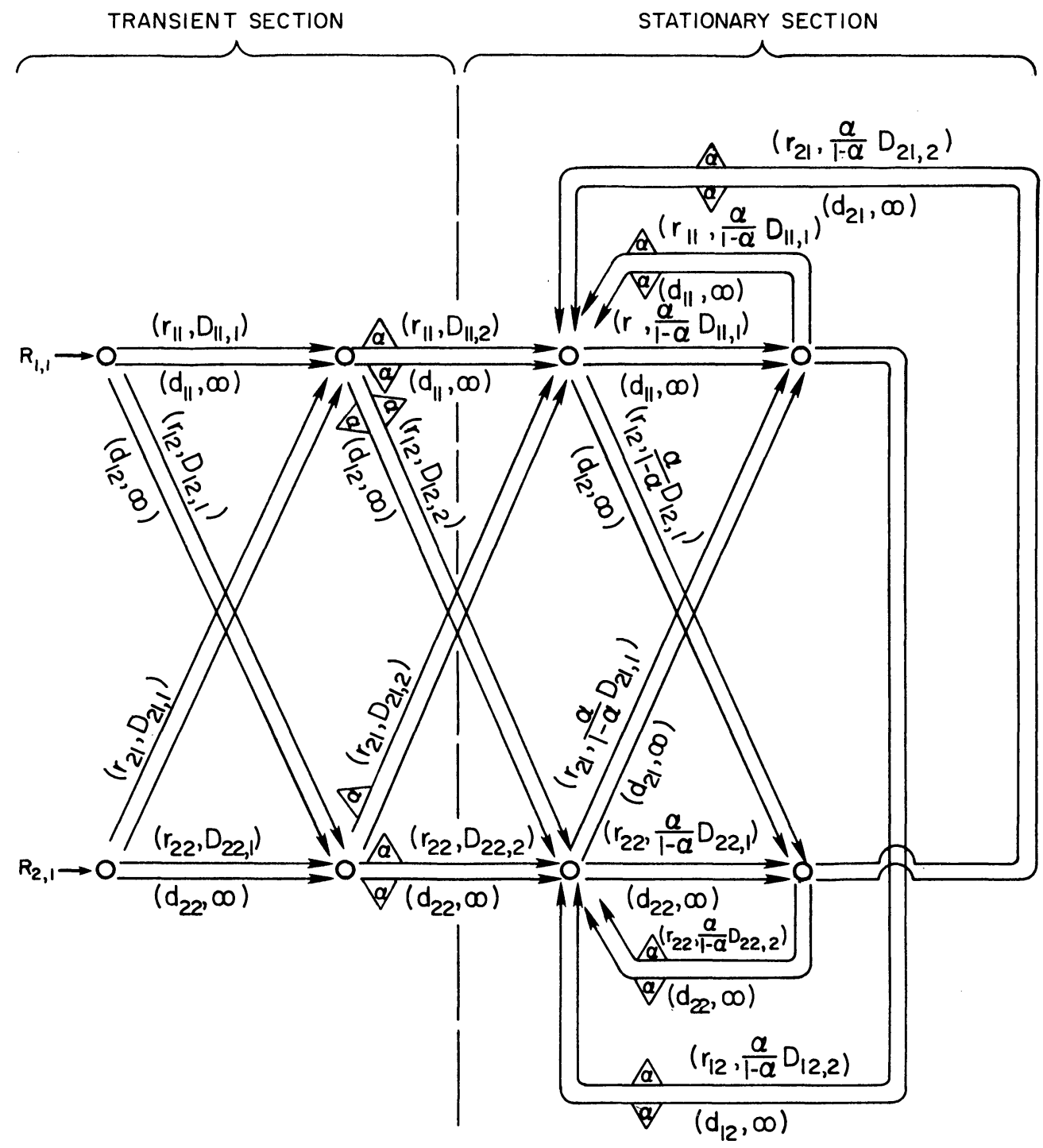

FIGURE 3. Network Representation of Generalized Summation Method.

$$
\sum_{t=0}^{\infty} \alpha^{t} \mathbf{c}_{t} \mathbf{x}_{t}=\mathbf{c}_{0} \mathbf{x}_{0}+\mathbf{c x}_{g s}(\alpha)
$$

The dual equilibrium method, however, aggregates the stationary section decision variable into $\mathbf{x}(\alpha)=(1-\alpha) \sum_{t=1}^{\infty} \alpha^{t-1} \mathbf{x}_{t}$, producing the objective function $\mathbf{c}_{0} \mathbf{x}_{0}+\alpha \mathbf{c x}(\alpha) /(1$ $-\alpha)$. Note that the cost coefficient vector $\mathbf{c}$ is factored by $\alpha /(1-\alpha)$.

The difference in aggregation methods affects the upper bounds on links as well as the objective function. Suppose $\mathbf{x}_{t}=\mathbf{x}$ for $t \geq 1$. Then $\mathbf{x}_{g s}(\alpha)=\alpha \mathbf{x} /(1-\alpha)$. Therefore the link upper bounds in the aggregated stationary periods are scaled by $\alpha /(1-\alpha)$. In the dual equilibrium model, the aggregated stationary stage decision variable would be

$$
\mathbf{x}(\alpha)=(1-\alpha) \sum_{t=1}^{\infty} \alpha^{t-1} \mathbf{x}_{t}=\mathbf{x} .
$$

Therefore the upper bounds of the arcs in the stationary section of the dual equilibrium method are not modified. 


\section{Leontief Approximation}

The Leontief approximation technique is a direct application of theory developed in Grinold and Hopkins (1973). It is attractive because if a specific set of assumptions is fulfilled, we can obtain the optimal solution to the decision variables in the transient period rather than just an approximation. Unfortunately, the DVA model does not fulfill those requirements, but a modified version does.

The problem Grinold and Hopkins (1973) address is

$$
\min u=f_{0}\left(\mathbf{x}_{\mathbf{0}}\right)+\alpha \mathbf{c \mathbf { x } _ { 1 }}+\alpha^{2} \mathbf{c} \mathbf{x}_{\mathbf{2}}+\cdots+\alpha^{t} \mathbf{c} \mathbf{x}_{t}+\cdots
$$

subject to

$$
\begin{array}{ll}
\mathbf{A}_{\mathbf{0}} \mathbf{x}_{\mathbf{0}} & \leq \mathbf{0}, \\
\mathbf{H}_{\mathbf{1}} \mathbf{x}_{\mathbf{0}}+\mathbf{A} \mathbf{x}_{\mathbf{1}} & =\mathbf{b}_{\mathbf{1}}, \\
\mathbf{H}_{\mathbf{2}} \mathbf{x}_{\mathbf{0}}+\mathbf{K}_{\mathbf{1}} \mathbf{x}_{\mathbf{1}}+\mathbf{A} \mathbf{x}_{\mathbf{2}} & =\mathbf{b}_{\mathbf{2}}, \\
\vdots & \\
\mathbf{H}_{\mathbf{t}} \mathbf{x}_{\mathbf{0}}+\mathbf{K}_{\mathbf{t}-\mathbf{1}} \mathbf{x}_{\mathbf{1}}+\mathbf{K}_{\mathbf{t}-\mathbf{2}} \mathbf{x}_{\mathbf{2}}+\cdots+\mathbf{A} \mathbf{x}_{\mathbf{t}} & =\mathbf{b}_{\mathbf{t}}, \\
\quad & \\
\quad \mathbf{x}_{\mathbf{t}} \geq \mathbf{0} \quad(t=0,1,2, \cdots) . &
\end{array}
$$

The finite model proposed in Grinold and Hopkins (1973) is summarized in the following theorem.

THEOREM 2 (Grinold and Hopkins 1973). Define three problems, $R, R^{\prime}$, and $P$ :

(R) $\quad \min w=$ cy subject to

$\left(R^{\prime}\right) \quad \min w=\mathbf{e}^{\mathbf{T}} \mathbf{K y}$ subject to

$$
\begin{array}{r}
\mathbf{A}(\alpha) \mathbf{y}=\mathbf{e} \\
\mathbf{y} \geq \mathbf{0},
\end{array}
$$

$$
\begin{array}{r}
\mathbf{A}(\alpha) \mathbf{y}=\mathbf{e}, \\
\mathbf{y} \geq \mathbf{0},
\end{array}
$$

$(P) \quad \min u=\mathbf{c x}_{\mathbf{0}}+\pi^{*} \mathbf{H} \mathbf{x}_{\mathbf{0}}-\pi^{*}(\alpha)$ subject to

$$
\begin{aligned}
\mathbf{A}_{\mathbf{0}} \mathbf{x}_{\mathbf{0}} & =\mathbf{0}, \\
\mathbf{x}_{\mathbf{0}} & \geq \mathbf{0},
\end{aligned}
$$

where $\mathbf{e}$ is a column of ones and $\pi^{*}$ is the optimal solution to the dual of $(R)$. If $\pi^{*}$ is nonpositive and $\mathbf{A}, \mathbf{K}$, and $\mathbf{A}(\alpha)$ are Leontief, then $P$ gives the optimal solution to the transient period of the infinite horizon problem, $\mathbf{x}_{\mathbf{0}}$. If at least one element of $\pi^{*}$ is positive, then $R^{\prime}$ and $R$ must both have optimal solutions for $\mathbf{x}_{0}$ in $P$ to be optimal in the infinite horizon problem.

The crucial assumption in Grinold and Hopkins (1973) is that the matrices A and $\mathbf{A}(\alpha)$ be Leontief substitution matrices. A Leontief substitution matrix has the property that there is exactly one positive element in each column, and the rest of the entries in each column are nonpositive. Thus, $\mathbf{A}$ is not a Leontief substitution matrix, because each column corresponding to a loaded movement has two positive entries. One represents the arc's origin and the other the upper bound. This suggests approximating the DVA problem by ignoring the upper bound on the revenue links. Mathematically, dropping the upper bounds consists of defining $\mathbf{A}=\mathbf{A}_{f}$ and redefining $\mathbf{c}$ and $\mathbf{b}_{\mathbf{t}}$ accordingly. To show that such a procedure produces a Leontief constraint matrix, we have the following theorem. 
THEOREM 3. $\quad \mathbf{A}_{\mathbf{f}}$ is a Leontief substitution matrix if vehicles are allowed to depart from each region at each time period.

Proof. By definition, $\mathbf{A}_{\mathbf{f}}$ is a Leontief substitution matrix if and only if each column of $\mathbf{A}_{\mathbf{f}}$ has exactly one positive element and there exists a nonnegative linear combination of the columns which is strictly positive. First note that in an uncapacitated DVA network there are only two nonzero entries in each column, which are of opposite sign. A positive element represents the origin of an arc; a negative one indicates the termination of an arc. Thus all arcs in the model have a positive entry in the corresponding column of $\mathbf{A}_{\mathbf{f}}$. The first criterion of a Leontief substitution matrix is satisfied. Now it must be shown that there exists an $x$ such that $\mathbf{A}_{\mathbf{f}} \mathbf{x}>\mathbf{0}$. Since $\mathbf{A}_{\mathbf{f}} \mathbf{x}=\mathbf{b}$ is merely the conservation of flow equations for each time stage (Flow out - Flow in = Net supply of vehicles), we can interpret the condition $\mathbf{A}_{\mathbf{f}} \mathbf{x}>\mathbf{0}$ in a physical sense. The condition $\mathbf{A}_{\mathbf{f}} \mathbf{x}=\mathbf{e}, \mathbf{x} \geq \mathbf{0}$ means that there is a set of nonnegative vehicle flows in which one vehicle is added at each node of the DVA network. By assumption, vehicles can leave any node in the network. Moreover, the network is acyclic since vehicles cannot move backward in time. Thus the extra vehicle introduced at the arbitrary node can move forward in the network. As the vehicle reaches a new node in the network, it can always find another arc to leave on to move forward in time. For convenience, define the vector $\mathbf{x}^{\mathbf{k}}$ as

$$
\mathbf{x}_{j}^{i}= \begin{cases}1 & \text { if the vehicle introduced at node } i \text { travels on link } j \\ 0 & \text { otherwise. }\end{cases}
$$

Since all the arcs are uncapacitated, the feasibility of a vehicle's path through time is unaffected by the introduction of other vehicles. Thus a vehicle can be introduced at each node in the network to produce a series of paths $\mathbf{x}^{1}, \ldots, \mathbf{x}^{\mathbf{k}}(k$ is twice the product of $N$ and the cardinality of $C$ ). Then let $x_{j}=\sum_{\mathbf{i}} \mathbf{x}_{\mathbf{j}}^{\mathbf{j}}$. Clearly $\mathbf{x} \geq \mathbf{0}$. Moreover, $\mathbf{A}_{\mathbf{f}} \mathbf{x}=\mathbf{e}$ since $x$ has been constructed by adding one vehicle at each node in the network. Finally, since there are finitely many nodes in (15)-(17), $x$ is bounded. Hence $A_{f}$ is a Leontief substitution matrix.

If the DVA model is modified so that the arcs in the stationary period are uncapacitated, the results of Grinold and Hopkins (1973) can be used. That is, define $\mathbf{K}_{t}, \mathbf{A}, \mathbf{x}, \mathbf{c}$ and $\mathbf{b}$ to be the same as in the dual equilibrium method, but modified to eliminate the upper bounds on the revenue links. The relevant quantities for the two-region example then become

$$
\begin{aligned}
& \text { First Period }
\end{aligned}
$$

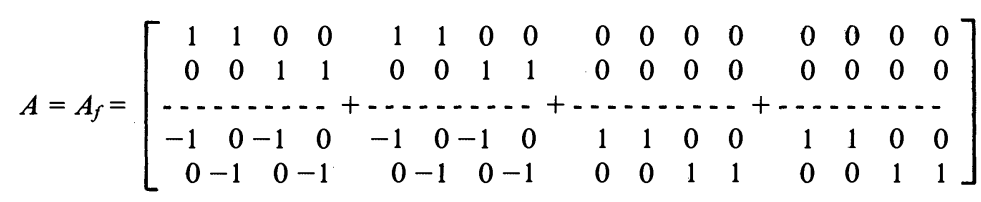

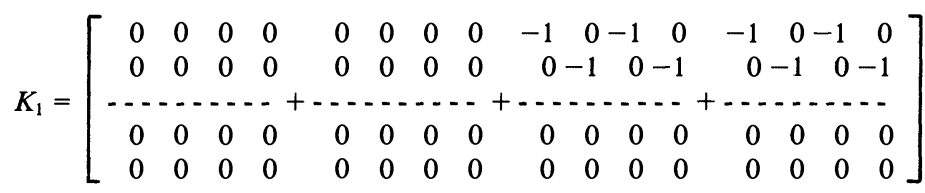

Full Empty

\begin{tabular}{c} 
Second Period \\
Full \\
\hline
\end{tabular}


We redefine the discounted sum of these matrices, $\mathbf{A}(\alpha)$, as

$$
\begin{gathered}
A(\alpha)=\left[\begin{array}{rrrrrrrrrrrrrrrr}
1 & 1 & 0 & 0 & 1 & 1 & 0 & 0 & -\alpha & 0 & -\alpha & 0 & -\alpha & 0 & -\alpha & 0 \\
0 & 0 & 1 & 1 & 0 & 0 & 1 & 1 & 0 & -\alpha & 0 & -\alpha & 0 & -\alpha & 0 & -\alpha \\
-1 & 0 & -1 & 0 & -1 & 0 & -1 & 0 & 1 & 1 & 0 & 0 & 1 & 1 & 0 & 0 \\
0 & -1 & 0 & -1 & 0 & -1 & 0 & -1 & 0 & 0 & 1 & 1 & 0 & 0 & 1 & 1
\end{array}\right], \\
\mathbf{c}=\left[-r_{11}-r_{12}-r_{21}-r_{22} d_{11} d_{12} d_{21} d_{22}-r_{11}-r_{12}-r_{21}-r_{22} d_{11} d_{12} d_{21} d_{22}\right], \\
\mathbf{b}_{t}=\mathbf{b}(\alpha)=[00]^{T}, \quad t>0 .
\end{gathered}
$$

The Leontief approximation to the DVA problem consists of removing the upper bounds on the loaded movement variables and solving problem $(R)$. If $\pi^{*}$ has no positive component, then solve $(P)$ to get $\mathbf{x}_{\mathbf{0}}$; otherwise, solve $\left(R^{\prime}\right)$. If some $\pi^{*}>0$ and $\left(R^{\prime}\right)$ has a bounded optimal solution, $(P)$ can be solved to get the infinite horizon optimal $\mathbf{x}_{\mathbf{0}}$. The actual implementation of this approximation technique is similar to that of the dual equilibrium procedure, for both require solving a generalized network. That is, Theorem 2 also ensures that the supplementary problem is a generalized network. Figure 4 presents the network corresponding to the constraint set for problems $R$ and $R^{\prime}$. The only difference between figure 4 and problem $R^{\prime}$ is the link costs, which are zero for forward pointing arcs and unity for backward pointing arcs. The dual

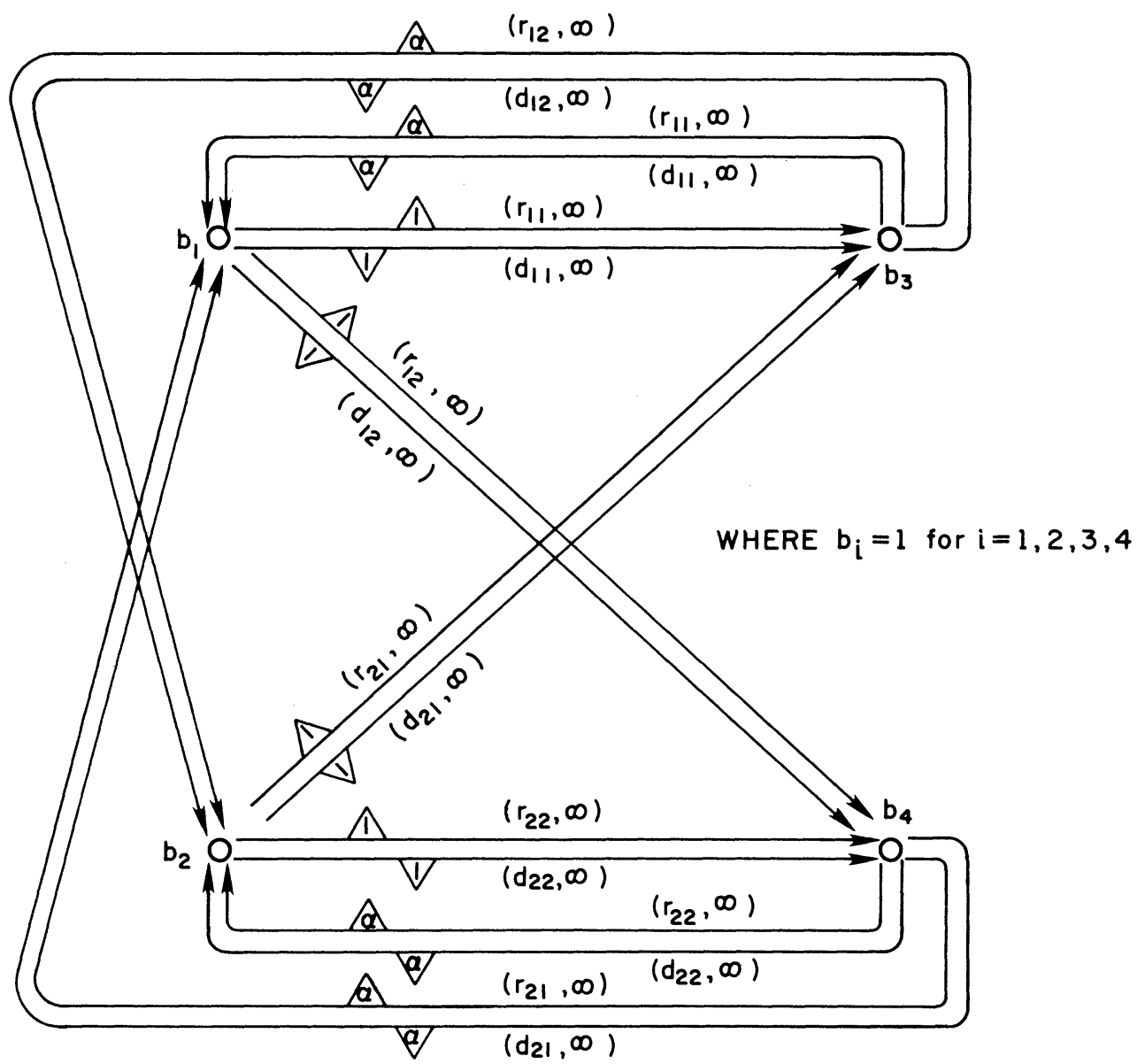

FIGURE 4. Network Representation of Leontief Approximation. 
variables for the first period of $(R)$ are then used as regional penalties for the truncated DVA model. That is, the dual for region $i$ is used as the cost for the arcs leaving region $i$ nodes for the supersink of the finite horizon DVA model.

Note that the economic interpretation of this "uncapacitated" DVA model is that there is infinite demand for vehicles between regions in the stationary period. Therefore the Leontief approach is unable to fully exploit the power of the results in Grinold and Hopkins (1973), since the problem being solved is only an approximation to the DVA model. If the DVA model being considered has very high demands on the whole, however, the Leontief model may serve as a good approximation technique.

\section{Numerical Experiments}

Each approximation method needs to be evaluated on how close its solution is to the infinite horizon model solution, as well as its computational requirements. This should be done in the context of practical usefulness rather than theoretical elegance. The tradeoffs each method faces are between computer requirements and accuracy relative to the true solution to the infinite horizon DVA model. The tradeoff is an important one. In actual implementations, the DVA model frequently must be run in real-time, often on an already heavily congested computer, and response times must be extremely fast. This section begins by introducing three new approximation methods to be used in judging the approximation techniques of the previous section. It then describes the method of measuring how "good" an approximate solution is, and it finishes by describing the experimental design and analyzing the numerical results.

\section{Brute Force Approximation}

For the purpose of evaluating the accuracy of different approximations, each problem tested is solved using a relatively long planning horizon, depending on the value of the discount factor $\alpha$. At the end of the planning horizon, all future movements are ignored. The solution of this problem is then assumed to be a reasonable approximation of the original infinite stage planning problem. Comparisons of solutions obtained by different approximations are then made using the flows during the transient stage.

The number of stages $N$ to be used in the brute force method is determined by finding the smallest integer $N$ such that $\alpha^{N} \leq \epsilon$, where $\epsilon$ is a suitably chosen tolerance parameter. In the experiments reported here, two values of $\alpha$ are used, with a different value of $\epsilon$ for each discount factor. Runs with $\alpha=0.3$ use $\epsilon=0.05(N=3)$, and runs with $\alpha=0.6$ use $\epsilon=0.02(N=8)$. Tests with smaller values of $\epsilon$ indicate that the first stage solution of the brute force method is not very sensitive to changes in $\epsilon$ below these levels.

\section{Naive Approach}

At the other extreme from the brute force method is the simplistic method of truncating the DVA model at the end of the first stage. The links corresponding to the vehicle movements terminating beyond the end of the planning horizon terminate at a supersink. That method should provide a bound on how "bad" an approximation can be relative to the brute force surrogate for the infinite horizon solution. Thus, if an approximation method performs "worse" than the naive approach it should be discarded for two reasons. First, the naive method is the simplest approximation procedure. Second, it is quick to solve because efficient network simplex codes designed for networks without gains or losses can be used.

\section{Naive Penalty Method}

It is clear that the basic naive method can be made to look arbitrarily bad by constructing attractive links into very unattractive areas near the end of the planning 
horizon. A natural way to help overcome the obvious problems of this approach is to use the naive solution to calculate regional end of period "penalties." Regional penalties are costs which attempt to capture the relative value of an additional vehicle in each region at the end of the first stage. The regional penalties are placed on the links destined for the supersink to give a modified one-stage DVA model. That modified model is solved and regional prices are calculated and re-applied to the links destined for the supersink. Thus the naive penalty model is iterative, and the modeller decides how many iterations to use.

The penalty $p(i ; n)$ is an approximation to the future worth of a vehicle at region $i$ in period $n$. Thus $p(i ; n)$ depends on where the vehicles are sent after being in region $i$ at time $n$. A vehicle that leaves region $i$ at time $n$ and arrives at $j$ at $n+t_{i j}$ contributes $r_{i j, n}$ (the revenue gained) or $d_{i j, n}$ (deadhead cost incurred) plus the future worth of being in region $j$ at $n+t_{i j}, p\left(j ; n+t_{i j}\right)$. Thus the regional penalties are calculated recursively by period, beginning at the end of the planning horizon and moving backward in time. The recursion formula for period $n$ is

$p(i ; n)=\left(\sum_{k \in C} F_{i k, n}+E_{i k, n}\right)^{-1} \sum_{j \in C}\left[F_{i j, n}\left(r_{i j, n}+p\left(i ; n+t_{i j}\right)\right)+E_{i j, n}\left(d_{i j, n}+p\left(i ; n+t_{i j}\right)\right)\right]$.

For the first iteration the penalties beyond the planning horizon of the naive solution are zero. For subsequent iterations of the method, the penalties beyond the planning horizon are the first period penalties from the previous iteration. That is, the cost on the link going from $i$ at the last time period into the supersink is taken to be $p(i ; 1)$. Again, since the network problem used for each iteration does not have any gains or losses, a specialized simplex code can be used to solve each iteration efficiently. In fact, because only a subset of objective function coefficients change from iteration to iteration, the old basis can be used as a starting point for the next iteration. These features make this method quick and simple. Therefore, alternative approximation methods should come closer to the brute force approximation than the naive penalty approach to be considered for use.

\section{Method of Measurement}

A "bad" approximation technique is one that provides a solution that does not agree well with the corresponding section of the optimal brute force solution. The measurement of "agreement" used here is the fraction of vehicles dispatched in a manner different from the brute force approach. This can be defined more precisely. Let $V_{n}$ be the number of vehicles available for dispatch during period $n$. Let $\hat{F}_{i j, n}$ and $\hat{E}_{i j, n}$ be the number of full and empty vehicles, respectively, that depart in period $n$ from region $i$ to region $j$ of the brute force model. Then the measure of deviation from the brute force solution during period $n$ is

$$
\delta_{n}=\left(2 V_{n}\right)^{-1}\left[\sum_{i \in C} \sum_{j \in C}\left|\left(\hat{F}_{i j, n}-F_{i j, n}\right)\right|+\left|\left(\hat{E}_{i j, n}-E_{i j, n}\right)\right|\right]
$$

where $\delta_{n}$ is interpreted as the fraction of vehicles originating in time period $n$ which are dispatched differently from the brute force solution. The results reported here are for the first period, which is of the most importance to a dispatcher using a DVA model, and the average deviation over the course of the $T$ period transient period. The average deviation statistic is $\delta_{T S}=T^{-1} \sum_{i=1}^{T} \delta_{i}$.

\section{Experimental Design}

The purpose of the numerical experiments is to examine the performance of the approximation techniques of $\S 3$ on a wide variety of test problems. The experimental design is thus based on different combinations of four parameters: the ratio of average 
demand per period to the number of vehicles in the system (termed here "level of demand"), the number of periods per time stage, the structure of the demands, and the weekly discount rate.

The test problems are created using a computer random number generator. The problem generator considers the regions to be points, located in a 1000 by 2000 mile rectangle. The $x$ and $y$ coordinates are randomly generated from uniform distributions, and the distances between points can be calculated by assuming a straight line path. To compute how many periods it requires to move between regions, the program computes the euclidean distance between the regions and uses the average speed (42 miles per hour) to find the average travel time. The deadhead and revenue figures for origin destination pairs are calculated by assuming one dollar per mile for empty deadhead costs and 35 cents per mile net revenue for loaded vehicles.

As the Alaska example of the introduction pointed out, end effects should be the most severe when there are regions with a high demand for vehicles into them but not very high demand for vehicles leaving them. The problem generator captures this by randomly generating "attraction potentials" and "generation potentials" for each region. The attraction and generation potentials are appropriately scaled numbers that are a measure of a region's ability to attract and generate flow, respectively. The product of the attraction potential of a destination of a link and the generation potential of the origin region, rounded to the nearest integer, is the expected demand, i.e. the upper bound on the link between those two regions.

Both the attraction and generation potentials are drawn from a uniform distribution on the unit interval. They are then scaled to make the average total demand per period equal to a specified fraction of the number of vehicles in the system, the "level of demand." One set of test problems draws the attraction and generation potentials independently. Hence this set is said to have "uncorrelated" demands. Another set of test problems are based on sampling the regional attraction potentials, $a_{i}$, and then calculating the generation potentials, $g_{i}$, by the equation $g_{i}=1-a_{i}$. This is the "correlated" demand set of problems. The motivation for this design is that myopic algorithms should perform relatively poorly using correlated demand and production factors, since this increases the likelihood of drawing a vehicle into a region with a high attraction potential, only to find little demand out of the region.

The test problems are designed to test a set of hypotheses about how the approximation methods perform. Of course the most important hypothesis to consider is that the dual equilibrium, generalized summation, and Leontief approximations are better than the naive and naive penalty methods. Beyond that, there is the question of how these methods compare with each other, and how the structure of the problem affects performance. Toward this goal, a series of experiments are run to test the affect of the level of demand, the discount rate, the number of time periods in each stage, the number of regions, and whether or not attraction and production potentials are correlated. The purpose of testing these variables is as follows:

- Level of demand-One of the key approximations is the aggregation of upper bounds (the demands). The quality of the solutions should all improve as the demands are increased relative to the supply of vehicles. In particular, the Leontief approximation should provide an exact solution when the upper bounds are infinite.

- Discount rate-As $\alpha$ is decreased, myopic algorithms such as the naive approach should perform better. As $\alpha$ is increased, the accuracy of the dual equilibrium and generalized summation procedures, as well as the relative computational efficiency over the brute force approach, should improve.

- Number of regions-The relative value of the more rigorous approaches may be more pronounced with larger problems, where there are more opportunities for making wrong decisions. 
- Number of time periods in each stage-The deviations from the brute force solution for the myopic formulations should be more pronounced when the number of time periods per stage is decreased, as this has the effect of shortening the planning horizon.

- Correlation of attraction and production potentials-The deviations from the brute force solution should increase for all approximation methods when the attraction and generation potentials are calculated using the equation $g_{i}=1-a_{i}$.

\section{Numerical Results}

Three test problems are created for each setting of parameters using the random problem generator. Since 34 different combinations of parameter settings are tested, 102 test problems are considered. Tables 1 and 2 give averages of the $\delta_{1}$ and $\delta_{T S}$ statistics, respectively. Each row represents the average of the test statistics for the three test problems at the parameter settings described by columns 1 through 6 . Tables 3 and

TABLE 1

$\delta_{1}$ Measure of Deviation from Brute Force Solution (each row is the average of three data points)

\begin{tabular}{|c|c|c|c|c|c|c|c|c|c|c|}
\hline $\begin{array}{l}\text { Level of } \\
\text { Demand }\end{array}$ & $\begin{array}{c}\text { Number } \\
\text { of } \\
\text { Regions }\end{array}$ & $\begin{array}{c}\text { Periods } \\
\text { per } \\
\text { Stage }\end{array}$ & $\begin{array}{c}\text { Cor- } \\
\text { related } \\
\text { Demands } \\
? \\
\end{array}$ & $\begin{array}{c}\text { Discount } \\
\text { Rate }\end{array}$ & $\begin{array}{c}\text { Planning } \\
\text { Horizon } \\
\text { of Brute } \\
\text { Force } \\
\text { Method }\end{array}$ & Naive & $\begin{array}{c}\text { Naive } \\
\text { Penalty }\end{array}$ & $\begin{array}{c}\text { Dual } \\
\text { Eq. }\end{array}$ & $\begin{array}{l}\text { Gen. } \\
\text { Sum. }\end{array}$ & $\begin{array}{l}\text { Leontief } \\
\text { Approx. }\end{array}$ \\
\hline 0.5 & 20 & 2 & yes & 0.600 & 8 & 0.107 & 0.267 & 0.052 & 0.045 & 0.142 \\
\hline 0.5 & 20 & 2 & yes & 0.300 & 3 & 0.082 & 0.264 & 0.010 & 0.013 & 0.133 \\
\hline 0.5 & 20 & 2 & no & 0.600 & 8 & 0.131 & 0.124 & 0.059 & 0.052 & 0.124 \\
\hline 0.5 & 20 & 2 & no & 0.300 & 3 & 0.099 & 0.114 & 0.006 & 0.008 & 0.110 \\
\hline 0.5 & 20 & 7 & yes & 0.300 & 3 & 0.097 & 0.097 & 0.104 & 0.145 & 0.122 \\
\hline 0.5 & 20 & 7 & no & 0.300 & 3 & 0.121 & 0.121 & 0.117 & 0.144 & 0.170 \\
\hline 0.5 & 39 & 2 & yes & 0.600 & 8 & 0.111 & 0.456 & 0.082 & 0.080 & 0.161 \\
\hline 0.5 & 39 & 2 & yes & 0.300 & 3 & 0.066 & 0.412 & 0.015 & 0.014 & 0.140 \\
\hline 0.5 & 39 & 2 & no & 0.600 & 8 & 0.113 & 0.182 & 0.076 & 0.060 & 0.162 \\
\hline 0.5 & 39 & 2 & no & 0.300 & 3 & 0.083 & 0.184 & 0.022 & 0.026 & 0.143 \\
\hline 1. & 20 & 2 & yes & 0.600 & 8 & 0.133 & 0.238 & 0.043 & 0.062 & 0.207 \\
\hline 1. & 20 & 2 & yes & 0.300 & 3 & 0.092 & 0.232 & 0.007 & 0.006 & 0.197 \\
\hline 1. & 20 & 2 & no & 0.600 & 8 & 0.141 & 0.178 & 0.066 & 0.060 & 0.176 \\
\hline 1. & 20 & 2 & no & 0.300 & 3 & 0.104 & 0.170 & 0.015 & 0.017 & 0.138 \\
\hline 1. & 20 & 7 & yes & 0.300 & 3 & 0.089 & 0.067 & 0.023 & 0.081 & 0.086 \\
\hline 1. & 20 & 7 & no & 0.300 & 3 & 0.080 & 0.079 & 0.062 & 0.099 & 0.062 \\
\hline 1. & 39 & 2 & yes & 0.600 & 8 & 0.122 & 0.429 & 0.055 & 0.057 & 0.281 \\
\hline 1. & 39 & 2 & yes & 0.300 & 3 & 0.056 & 0.425 & 0.036 & 0.036 & 0.232 \\
\hline 1. & 39 & 2 & no & 0.600 & 8 & 0.122 & 0.244 & 0.073 & 0.078 & 0.205 \\
\hline 1. & 39 & 2 & no & 0.300 & 3 & 0.074 & 0.231 & 0.029 & 0.025 & 0.162 \\
\hline 10 & 20 & 2 & yes & 0.600 & 8 & 0.162 & 0.155 & 0.028 & 0.027 & 0.197 \\
\hline 10 & 20 & 2 & yes & 0.300 & 3 & 0.125 & 0.133 & 0.002 & 0.005 & 0.173 \\
\hline 10 & 20 & 2 & no & 0.600 & 8 & 0.115 & 0.170 & 0.063 & 0.063 & 0.210 \\
\hline 10 & 20 & 2 & no & 0.300 & 3 & 0.090 & 0.165 & 0.033 & 0.033 & 0.207 \\
\hline 10 & 20 & 7 & yes & 0.300 & 3 & 0.058 & 0.068 & 0.003 & 0.000 & 0.040 \\
\hline 10 & 20 & 7 & no & 0.300 & 3 & 0.111 & 0.128 & 0.005 & 0.005 & 0.036 \\
\hline 10 & 39 & 2 & yes & 0.600 & 8 & 0.128 & 0.166 & 0.021 & 0.021 & 0.185 \\
\hline 10 & 39 & 2 & yes & 0.300 & 3 & 0.121 & 0.197 & 0.021 & 0.021 & 0.168 \\
\hline 10 & 39 & 2 & no & 0.600 & 8 & 0.075 & 0.182 & 0.035 & 0.033 & 0.150 \\
\hline 10 & 39 & 2 & no & 0.300 & 3 & 0.066 & 0.175 & 0.028 & 0.028 & 0.150 \\
\hline inf & 20 & 2 & yes & 0.600 & 8 & 0.000 & 0.363 & 0.000 & 0.000 & 0.000 \\
\hline inf & 20 & 2 & yes & 0.300 & 3 & 0.000 & 0.142 & 0.000 & 0.000 & 0.020 \\
\hline inf & 39 & 2 & no & 0.600 & 8 & 0.064 & 0.370 & 0.000 & 0.000 & 0.056 \\
\hline inf & 39 & 2 & no & 0.300 & 3 & 0.016 & 0.282 & 0.016 & 0.016 & 0.114 \\
\hline \multicolumn{6}{|c|}{ All Problems } & 0.093 & 0.212 & 0.036 & 0.040 & 0.143 \\
\hline
\end{tabular}


TABLE 2

$\delta_{\mathrm{TS}}$ Measure of Deviation from Brute Force Solution (each row is the average of three data points)

\begin{tabular}{|c|c|c|c|c|c|c|c|c|c|c|}
\hline $\begin{array}{l}\text { Level of } \\
\text { Demand }\end{array}$ & $\begin{array}{c}\text { Number } \\
\text { of } \\
\text { Regions }\end{array}$ & $\begin{array}{c}\text { Periods } \\
\text { per } \\
\text { Stage }\end{array}$ & $\begin{array}{c}\text { Cor- } \\
\text { related } \\
\text { Demands } \\
? \\
\end{array}$ & $\begin{array}{c}\text { Discount } \\
\text { Rate }\end{array}$ & $\begin{array}{l}\text { Planning } \\
\text { Horizon } \\
\text { of Brute } \\
\text { Force } \\
\text { Method }\end{array}$ & Naive & $\begin{array}{c}\text { Naive } \\
\text { Penalty }\end{array}$ & $\begin{array}{c}\text { Dual } \\
\text { Eq. }\end{array}$ & $\begin{array}{l}\text { Gen. } \\
\text { Sum. }\end{array}$ & $\begin{array}{l}\text { Leontief } \\
\text { Approx. }\end{array}$ \\
\hline 0.5 & 20 & 2 & yes & 0.600 & 8 & 0.158 & 0.403 & 0.066 & 0.059 & 0.124 \\
\hline 0.5 & 20 & 2 & yes & 0.300 & 3 & 0.098 & 0.424 & 0.013 & 0.016 & 0.132 \\
\hline 0.5 & 20 & 2 & no & 0.600 & 8 & 0.150 & 0.247 & 0.076 & 0.066 & 0.148 \\
\hline 0.5 & 20 & 2 & no & 0.300 & 3 & 0.111 & 0.250 & 0.008 & 0.010 & 0.123 \\
\hline 0.5 & 20 & 7 & yes & 0.300 & 3 & 0.244 & 0.233 & 0.101 & 0.108 & 0.143 \\
\hline 0.5 & 20 & 7 & no & 0.300 & 3 & 0.261 & 0.259 & 0.107 & 0.119 & 0.170 \\
\hline 0.5 & 39 & 2 & yes & 0.600 & 8 & 0.155 & 0.671 & 0.095 & 0.100 & 0.189 \\
\hline 0.5 & 39 & 2 & yes & 0.300 & 3 & 0.091 & 0.656 & 0.020 & 0.020 & 0.147 \\
\hline 0.5 & 39 & 2 & no & 0.600 & 8 & 0.135 & 0.397 & 0.083 & 0.077 & 0.168 \\
\hline 0.5 & 39 & 2 & no & 0.300 & 3 & 0.086 & 0.407 & 0.024 & 0.026 & 0.130 \\
\hline 1. & 20 & 2 & yes & 0.600 & 8 & 0.190 & 0.403 & 0.060 & 0.077 & 0.230 \\
\hline 1. & 20 & 2 & yes & 0.300 & 3 & 0.112 & 0.432 & 0.009 & 0.008 & 0.180 \\
\hline 1. & 20 & 2 & no & 0.600 & 8 & 0.155 & 0.241 & 0.067 & 0.061 & 0.185 \\
\hline 1. & 20 & 2 & no & 0.300 & 3 & 0.102 & 0.252 & 0.016 & 0.018 & 0.131 \\
\hline 1. & 20 & 7 & yes & 0.300 & 3 & 0.212 & 0.204 & 0.042 & 0.054 & 0.152 \\
\hline 1. & 20 & 7 & no & 0.300 & 3 & 0.194 & 0.183 & 0.041 & 0.050 & 0.097 \\
\hline 1. & 39 & 2 & yes & 0.600 & 8 & 0.204 & 0.608 & 0.080 & 0.083 & 0.304 \\
\hline 1. & 39 & 2 & yes & 0.300 & 3 & 0.082 & 0.631 & 0.039 & 0.039 & 0.211 \\
\hline 1. & 39 & 2 & no & 0.600 & 8 & 0.191 & 0.329 & 0.095 & 0.106 & 0.237 \\
\hline 1. & 39 & 2 & no & 0.300 & 3 & 0.102 & 0.347 & 0.033 & 0.027 & 0.160 \\
\hline 10 & 20 & 2 & yes & 0.600 & 8 & 0.249 & 0.364 & 0.046 & 0.045 & 0.250 \\
\hline 10 & 20 & 2 & yes & 0.300 & 3 & 0.188 & 0.332 & 0.012 & 0.015 & 0.193 \\
\hline 10 & 20 & 2 & no & 0.600 & 8 & 0.169 & 0.410 & 0.059 & 0.060 & 0.234 \\
\hline 10 & 20 & 2 & no & 0.300 & 3 & 0.137 & 0.395 & 0.032 & 0.032 & 0.217 \\
\hline 10 & 20 & 7 & yes & 0.300 & 3 & 0.289 & 0.305 & 0.013 & 0.011 & 0.160 \\
\hline 10 & 20 & 7 & no & 0.300 & 3 & 0.294 & 0.325 & 0.010 & 0.011 & 0.130 \\
\hline 10 & 39 & 2 & yes & 0.600 & 8 & 0.268 & 0.401 & 0.026 & 0.026 & 0.286 \\
\hline 10 & 39 & 2 & yes & 0.300 & 3 & 0.215 & 0.431 & 0.020 & 0.020 & 0.230 \\
\hline 10 & 39 & 2 & no & 0.600 & 8 & 0.132 & 0.425 & 0.035 & 0.033 & 0.176 \\
\hline 10 & 39 & 2 & no & 0.300 & 3 & 0.123 & 0.420 & 0.027 & 0.027 & 0.170 \\
\hline inf & 20 & 2 & yes & 0.600 & 8 & 0.021 & 0.617 & 0.000 & 0.000 & 0.000 \\
\hline inf & 20 & 2 & yes & 0.300 & 3 & 0.000 & 0.571 & 0.000 & 0.000 & 0.020 \\
\hline inf & 39 & 2 & no & 0.600 & 8 & 0.064 & 0.685 & 0.000 & 0.000 & 0.056 \\
\hline inf & 39 & 2 & no & 0.300 & 3 & 0.030 & 0.641 & 0.016 & 0.016 & 0.117 \\
\hline \multicolumn{6}{|c|}{ All problems } & 0.153 & 0.111 & 0.040 & 0.041 & 0.165 \\
\hline
\end{tabular}

4 give averages by parameter level. Each row represents the average of the test problems with the parameter settings in column 1 .

Evaluating the results presented in Tables 1 through 4 is not just a matter of deciding which entry is smallest. Because of degeneracy, an approximation technique may provide an optimal solution, but one that does not match the optimal solution provided by the brute force approximation. To account for this, a set of tests is performed to estimate the variance of $\delta_{1}$ and $\delta_{T S}$. A variation of the dual equilibrium model is created by trivially changing the network structure. The resulting modified dual equilibrium structure gives the same optimal objective function value as the dual equilibrium method, although it usually produces a different transient stage solution as a result of network degeneracy. The difference between the two optimal solutions to the dual equilibrium problem can be used to estimate the variance of $\delta_{1}$ and $\delta_{T S}$ due to degener- 
TABLE 3

Average Deviations from the First Period of Brute Force Solution by Parameter Level

\begin{tabular}{lcccccc}
\hline $\begin{array}{c}\text { Parameter } \\
\text { Level }\end{array}$ & $\begin{array}{c}\text { Number of } \\
\text { Observations }\end{array}$ & Naive & $\begin{array}{c}\text { Naive } \\
\text { Penalty }\end{array}$ & $\begin{array}{c}\text { Dual } \\
\text { Equilibrium }\end{array}$ & $\begin{array}{c}\text { Generalized } \\
\text { Summation }\end{array}$ & $\begin{array}{c}\text { Leontief } \\
\text { Approx. }\end{array}$ \\
\hline LOD $=0.5$ & 30 & 0.1011 & 0.2221 & 0.0543 & 0.0587 & 0.1406 \\
LOD $=1$ & 30 & 0.1013 & 0.2292 & 0.0410 & 0.0520 & 0.1745 \\
LOD $=10$ & 30 & 0.1050 & 0.1538 & 0.0239 & 0.0240 & 0.1520 \\
LOD $=$ inf. & 12 & 0.0198 & 0.2192 & 0.0040 & 0.0040 & 0.0476 \\
Per. $=2$ & 84 & 0.0927 & 0.2374 & 0.0319 & 0.0316 & 0.1551 \\
Per. $=7$ & 18 & 0.0928 & 0.0933 & 0.0524 & 0.0791 & 0.0859 \\
Corr. $=$ Yes & 51 & 0.0911 & 0.2417 & 0.0294 & 0.0360 & 0.1461 \\
Corr. $=$ No. & 51 & 0.0944 & 0.1822 & 0.0415 & 0.0439 & 0.1397 \\
Alpha $=0.6$ & 42 & 0.1087 & 0.2517 & 0.0466 & 0.0456 & 0.1611 \\
Alpha $=0.3$ & 60 & 0.0816 & 0.1843 & 0.0277 & 0.0361 & 0.1301 \\
Regions $=20$ & 60 & 0.0969 & 0.1637 & 0.0349 & 0.0432 & 0.1274 \\
Regions $=39$ & 42 & 0.0869 & 0.2810 & 0.0364 & 0.0354 & 0.1650 \\
\hline
\end{tabular}

acy. The dual equilibrium model gives measures $\delta_{1}$ and $\delta_{T S}$ of deviation from the brute force method and the modified dual equilibrium model gives $\delta_{1}^{\prime}$ and $\delta_{T S}^{\prime}$. Thus the difference between their deviation from the brute force solution is $\nu_{1}=\delta_{1}-\delta_{1}^{\prime}$ and $\nu_{T S}$ $=\delta_{T S}-\delta_{T S}^{\prime}$. Note that $\delta_{1}, \delta_{1}^{\prime}, \delta_{T S}$ and $\delta_{T S}^{\prime}$ can be considered realizations of random variables since network flow models often have many optimal solutions from which the computer selects only one. Since the difference between two random variables is a random variable, $\nu_{1}$ and $\nu_{T S}$ are random variables. Forty-two of the test problems are used to generate the sets of results $\left\{\nu_{1}\right\}$ and $\left\{\nu_{T S}\right\}$. We first would like to test the hypothesis that the true means of $\nu_{1}$ and $\nu_{T S}$ are zero. Assuming the two quantities are normally distributed, which the numerical experiments indicate, a paired comparison Student $T$ test is used. The $t$-statistics for the hypotheses that the true means of $\nu_{1}$ and $\nu_{T S}$ are zero are 0.78 and 0.66 , respectively, indicating that it is safe to conclude that the actual means are zero. The variance is then calculated using zero as the true mean and 42 degrees of freedom. The estimates of the standard deviations of $\nu_{1}$ and $\nu_{T S}$ are 0.0334 and 0.0190 . These values should be kept in mind when evaluating the numerical results in the tables, since these values are estimates of the standard deviation of the variability in $\delta_{1}$ and $\delta_{T S}$ due to degeneracy.

TABLE 4

Average Deviations from the Transient Period of the Brute Force Solution by Parameter Level

\begin{tabular}{lcccccc}
\hline $\begin{array}{c}\text { Parameter } \\
\text { Level }\end{array}$ & $\begin{array}{c}\text { Number of } \\
\text { Observations }\end{array}$ & Naive & $\begin{array}{c}\text { Naive } \\
\text { Penalty }\end{array}$ & $\begin{array}{c}\text { Dual } \\
\text { Equilibrium }\end{array}$ & $\begin{array}{c}\text { Generalized } \\
\text { Summation }\end{array}$ & $\begin{array}{c}\text { Leontief } \\
\text { Approx. }\end{array}$ \\
\hline LOD $=0.5$ & 30 & 0.1011 & 0.2221 & 0.0543 & 0.0587 & 0.1406 \\
LOD $=1$ & 30 & 0.1013 & 0.2293 & 0.0409 & 0.0520 & 0.1745 \\
LOD $=10$ & 30 & 0.1050 & 0.1538 & 0.0239 & 0.0236 & 0.1516 \\
LOD $=$ inf. & 12 & 0.0199 & 0.2892 & 0.0040 & 0.0040 & 0.0476 \\
Per. $=2$ & 84 & 0.0928 & 0.2375 & 0.0318 & 0.0316 & 0.1551 \\
Per. $=7$ & 18 & 0.0928 & 0.0933 & 0.0524 & 0.0791 & 0.0858 \\
Corr. $=$ Yes & 51 & 0.0911 & 0.2418 & 0.0295 & 0.0361 & 0.1461 \\
Corr. $=$ No. & 51 & 0.0944 & 0.1823 & 0.0415 & 0.0440 & 0.1397 \\
Alpha $=0.6$ & 42 & 0.1088 & 0.2516 & 0.0466 & 0.0460 & 0.1611 \\
Alpha $=0.3$ & 60 & 0.0812 & 0.1843 & 0.0277 & 0.0361 & 0.1301 \\
Regions $=20$ & 60 & 0.0969 & 0.1637 & 0.0349 & 0.0432 & 0.1274 \\
Regions $=39$ & 42 & 0.0869 & 0.2869 & 0.0364 & 0.0354 & 0.1650 \\
\hline
\end{tabular}


Keeping this in mind, it is clear from Tables 1 and 2 that both the dual equilibrium and generalized summation methods perform better than the naive, naive penalty, and Leontief approximation methods. However, the dual equilibrium or generalized summation methods are effectively equivalent, as expected. The results of Tables 3 and 4 suggest that decreasing the number of regions in the model reduces the deviations from the brute force solution for all but the naive penalty and generalized summation methods; reducing the discount rate improves the performance of all methods over the brute force approach. However, considering the standard deviation due to degeneracy, it is safe to conclude that $\alpha$ and the number of regions only affect the naive penalty method and the Leontief Approximations. As expected, the higher levels of demand significantly improve the quality of the solutions, except for the naive and naive penalty methods. Moreover, the Leontief approximation performs much better at the infinite level of demand. At the three more realistic levels of demand, however, the Leontief approximation is outperformed even by the naive approach. Note that although theory suggests that the deviations from the Leontief approximation at infinite levels of demand should be zero, degeneracy produces positive deviations. The effect of using more periods per stage improves the quality of the naive penalty approach while impairing the performance of the dual equilibrium and generalized summation methods. Somewhat surprisingly, the intuitive effect of the correlation of attraction and generation potentials is not supported by the data.

It is important to note that the solutions given by the approximation methods which formulate as generalized networks contain fractional link flows in the transient period. Unlike pure networks, there is no guarantee of an integer optimal solution for generalized networks. To implement the dual equilibrium and generalized summation methods, then, would require some method for integerizing the solution. The appeal of the naive, naive penalty, and Leontief approximation methods is that they do not face this problem.

The final evaluation of the approximation techniques should consider not only accuracy but also computer requirements. In particular, practical implementation issues put a limit on the amount of computer time required to solve the DVA model. As such, the brute force solution places an upper bound on the amount of computer time required to solve the model. If an approximation method takes longer to solve than the brute force model, the user may as well use the brute force model instead. The software used for testing was written in FORTRAN 77, with the exception of the generalized network code (Mulvey and Zenios, 1985), which was written in FORTRAN IV. All programs were compiled using an optimizing compiler and were run on an IBM 3081. Typical runs times for 39 region problems were 11.3 seconds for the dual equilibrium method and 10.8 seconds for the generalized summation method. A typical run time for the brute force method was 34 seconds for a planning horizon of eight stages and 6 seconds for a planning horizon of three stages. The naive approach required 3 seconds. Thus the only two methods that provided better solutions than the naive approach, the dual equilibrium and generalized summation models, required more time to solve than the brute force method when $\alpha=0.3$ and the planning horizon was three stages long. Therefore the dual equilibrium and generalized summation models should be used unless $\alpha$ is relatively small, since a small discount factor implies a short brute force model that can be solved quickly.

\section{Conclusions}

We have shown that finite generalized network models can be used to approximate the first stage solution of the infinite horizon DVA model. Although such an approach to mitigating end effects in the DVA model worked well on the test problems consid- 
ered, it required the computational expense of solving generalized network problems. A goal of future research is to discover methods for reducing end effects in the DVA model which can be formulated as pure networks. Then more efficient solution techniques for networks without gains or losses on links such as Kennington and Helgason (1980) could be used. Moreover, such an approach would avoid the problem of fractional solutions.

\section{References}

$\rightarrow$ Brown, G. B. AND R. D. MCBRIDE, "Solving Generalized Networks," Management Sci., 30 (1984), 1497-1523.

GRINOLD, R., "Finite Horizon Approximations of Infinite Horizon Linear Programs," Math. Programming, 12 (1977), 1-17.

$\rightarrow-$, "Model Building Techniques for the Correction of End Effects in Multistage Convex Programs," Oper. Res., 31, 3 (1983a), 407-431.

—_, "Convex Infinite Horizon Programs," Math. Programming, 25 (1983b), 64-82.

$\longrightarrow$ AND D. S. P. HoPKINS, "Computing Optimal Solutions for Infinite-Horizon Mathematical Programs with a Transient Stage," Oper. Res., 21 (1973), 179-187.

Hopkins, D. S. P., "Infinite-Horizon Optimality in an Equipment Replacement and Capacity Expansion Model," Management Sci., 18 (1971), 145-156.

JoRdAN, W. C. AND M. A. TURNQuIST, "A Stochastic, Dynamic Network Model for Railroad Car Distribution," Transportation Sci., 17 (1983), 123-145.

Kennington, J. L., AND R. V. Helgason, Algorithms for Network Programming, Wiley, New York, 1980.

LEDDON, C. D. AND E. Wrathall, Scheduling Empty Freight Car Fleets on the Louisville and Nashville Railroad, Second International Symposium on the Use of Cybernetics on the Railways, Montreal, Canada, October 1-6, 1967.

MANNE, A. S., "Sufficient Conditions for Optimality in an Infinite Horizon Development Plan," Econometrica, 38 (1970), 18-38.

MulveY, J. M. AND S. ZeNios, “Solving Large Scale Generalized Networks,” J. Informat. Optim. Sci., 6 (1985), 95-112.

Potrs, R. B., "Movement of Empty Containers within Australia," Presented at Operations Research Society of Victoria, Melbourne, Australia, September 17, 1970.

Powell, W. B., "A Stochastic Formulation of the Dynamic Vehicle Allocation Problem," Transportation Sci., 20 (1986), 117-129.

- Y. SHEFFI ANDS. THIRIEZ, "The Dynamic Vehicle Allocation Problem with Uncertain Demands," in Ninth Internat. Sympos. Transportation and Traffic Theory, VNU Science Press, 1984, 357-374.

White, W. W., "Dynamic Transshipment Networks: An Algorithm and Its Application to the Distribution of Empty Containers," Networks, 2, 3 (1972), 211-236.

- AND A. M. Bomberault, "A Network Algorithm for Empty Freight Car Allocation," IBM Systems J., 8, 2 (1969), 147-171. 